American Journal of Applied Sciences 4 (4): 197-202, 2007

ISSN 1546-9239

(C) 2007 Science Publications

\title{
Crack Analysis of Concrete Arch Dams Using Micro-Planes Damage Based Constitutive Relations
}

\author{
M. Labibzadeh and S. A. Sadrnejad \\ Department of Civil Engineering, K.N. Toosi University of Technology, Tehran, Iran
}

\begin{abstract}
Among the various numerical simulating models of plane concrete, the micro-planes models have an excellent position. These models are not as complicated as microscopic models such as discrete particles models and do not have the shortcomings of macroscopic models based on the stress or strain invariants. The object of this study is the application of author's recently developed microplanes damage based model of plane concrete through a 3D finite elements code to show it's abilities in crack/damage analysis of actual concrete structures such as concrete double curvature arch dams. The proposed code not only able to predict the crack line, but also determine which combination of loading conditions occurs on damaged micro-planes. Validity of the proposed code is investigated through a few standard benchmark examples.
\end{abstract}

Keywords: Micro-planes theory, continuum damage theory, finite elements method, concrete arch dams

\section{INTRODUCTION}

Surveys through the continuum macroscopic constitutive models of concrete shows that micro-planes based models have an excellent place. In these models, instead of presenting constitutive relations in the shape of stress and strain tensors, the stress and strain vectors which are in turn the projections of stress or strain tensors are used. This method not only provides a more physical conceptual base for mind, but also leads to more simple mathematical formulation. On the other hand, invariant-based continuum macroscopic models lose some of the important features of material behavior because they are basically not able to capture and store the data properties in the different directions around a material point whereas the micro-planes models inherently include the directional characteristics of a material point.

Many of the available 3D commercial analyzing codes are not equipped with the proper concrete material model, so are not able to perform precisely crack/damage analysis of concrete structures.

The object of this study is the application of a proposed micro-planes damage based model of plane concrete $^{[1]}$ through a 3D finite elements code to show it's abilities in crack/damage analysis of concrete structures such as concrete double curvature arch dams. The proposed code not only able to predict the crack line, but also determine which combination of loading conditions occurs on damaged micro-planes. Validity of the proposed code is investigated through a few standard benchmark examples.

Concrete model: The proposed concrete model used in this study is a combination of micro-planes theory and continuum damage theory developed by the authors ${ }^{[1]}$. In this model the constitutive modulus matrix is computed from superposition of its counterparts on the micro-planes that such counterparts in turn, are calculated based on the damages occurred on each micro-plane during load history depending on its specific loading conditions ${ }^{[1]}$. This damage is evaluated according to the five separate damage functions; each of them belongs to the particular loading states. This five loading conditions are as follows:

I. hydrostatic compression

II. hydrostatic extension

III. pure shear

IV. shear + compression

V. shear + extension

On each micro-plane at each time of loading history, there exists one specific loading situation that it may be in one of the five mentioned basic loading conditions. For every five mood, a specific damage function according to the authoritative laboratory test results available in the literature is assigned. Then, for each state of on plane loading, one of the five introduced damage functions will be computed with respect to the history of micro-stress and strain components ${ }^{[1]}$.

Corresponding Author: $\quad$ M. Labibzadeh, Department of Civil Engineering, K.N. Toosi University of Technology, Tehran, Iran 


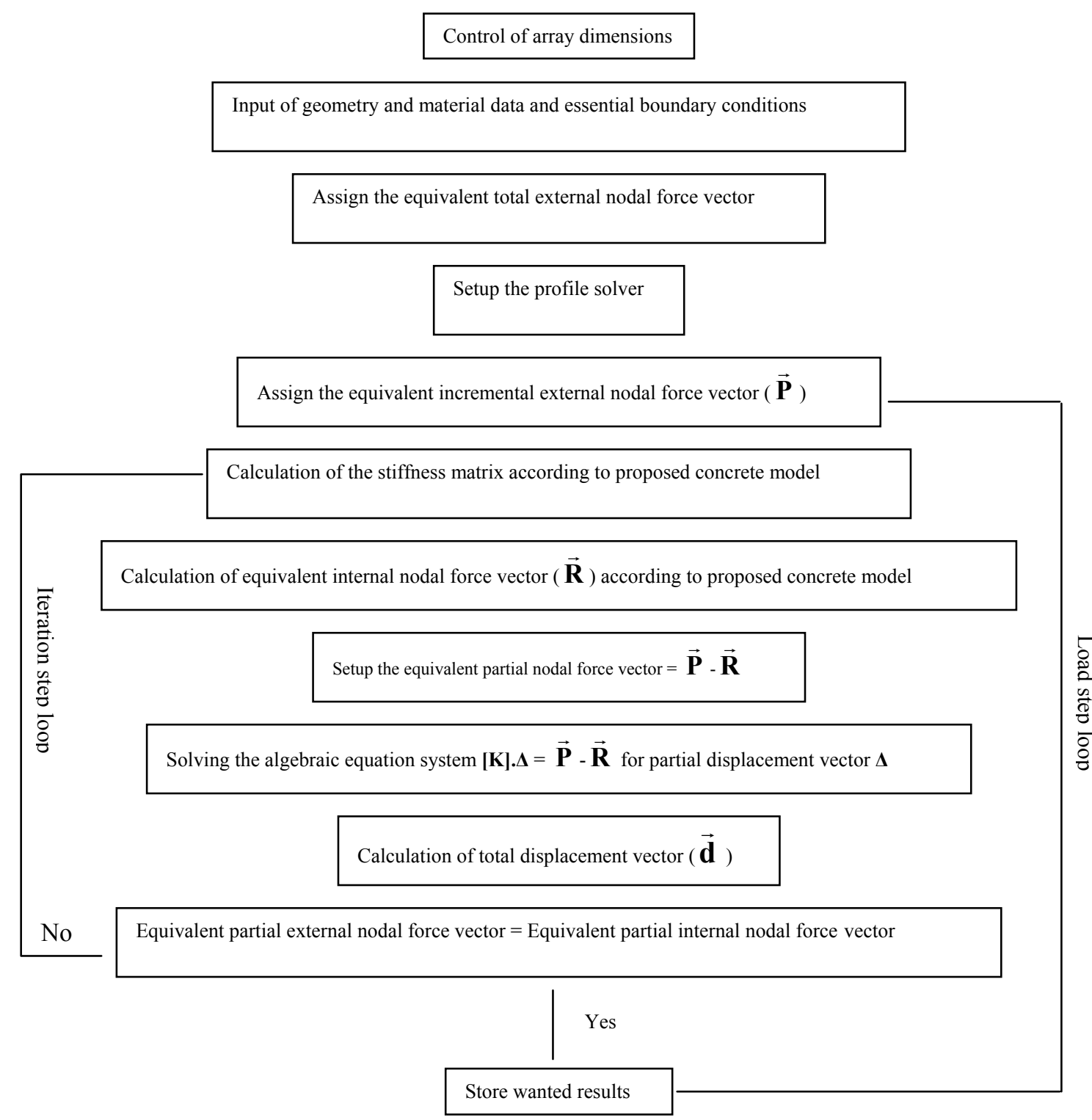

Fig. 1: Flowchart of proposed software in nonlinear static analysis
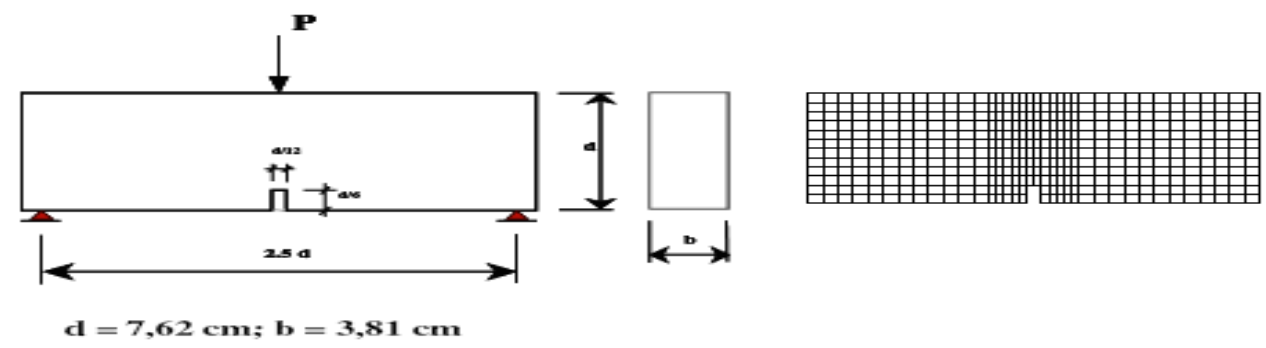

Fig. 2: Geometry and finite elements mesh of three-point-bending test 


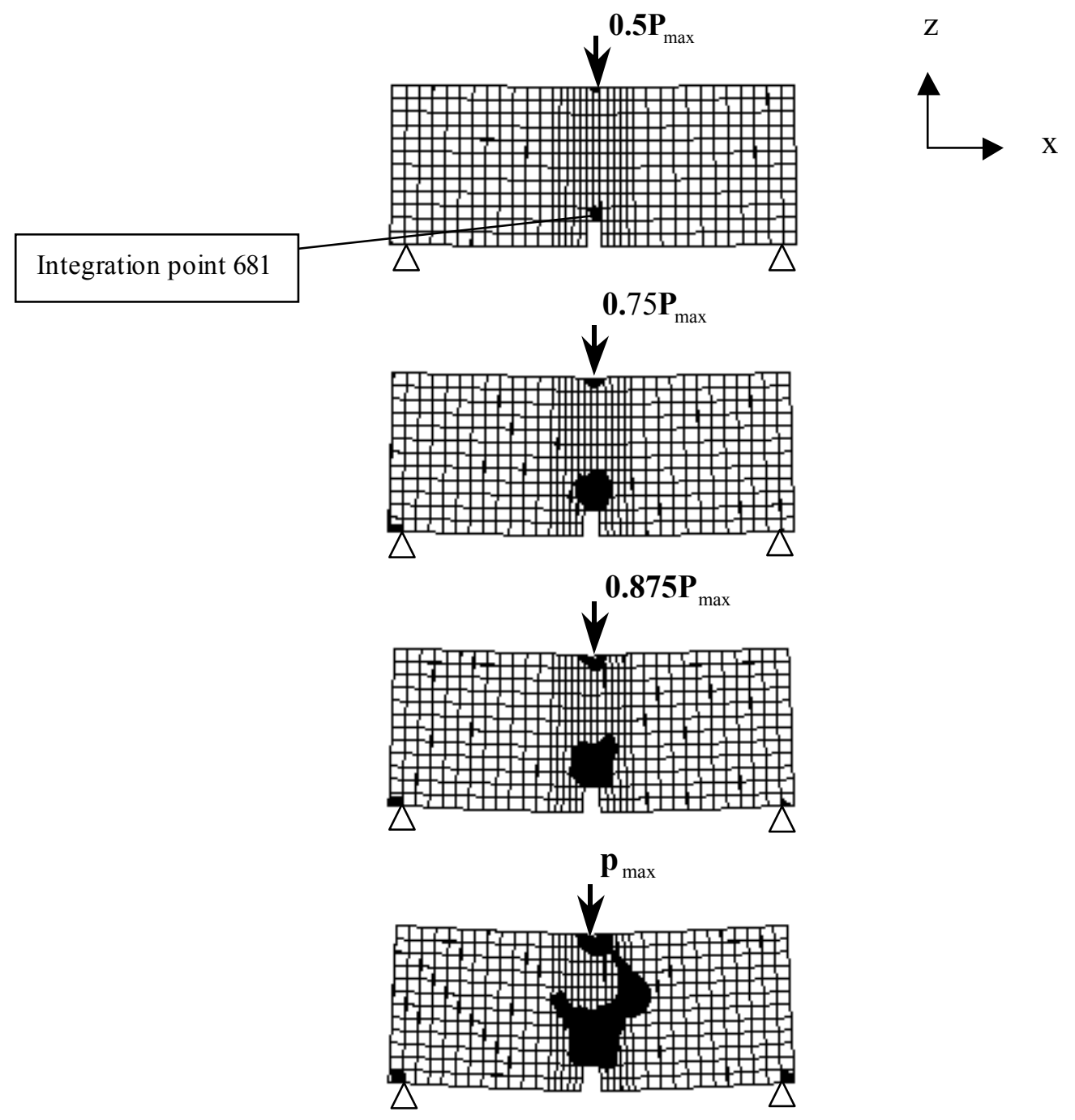

Fig. 3: Crack growth path and geometry changes of three-point-bending test

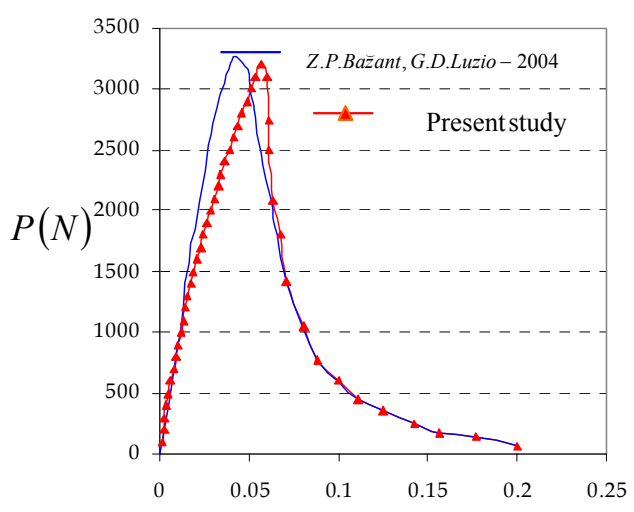

Fig.4: Load-displacement curve of three-pointbending test

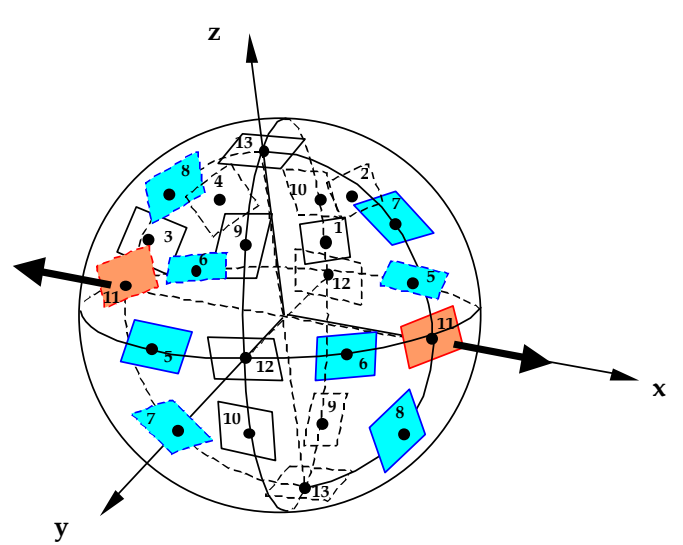

Fig. 5: Damaged micro-planes 


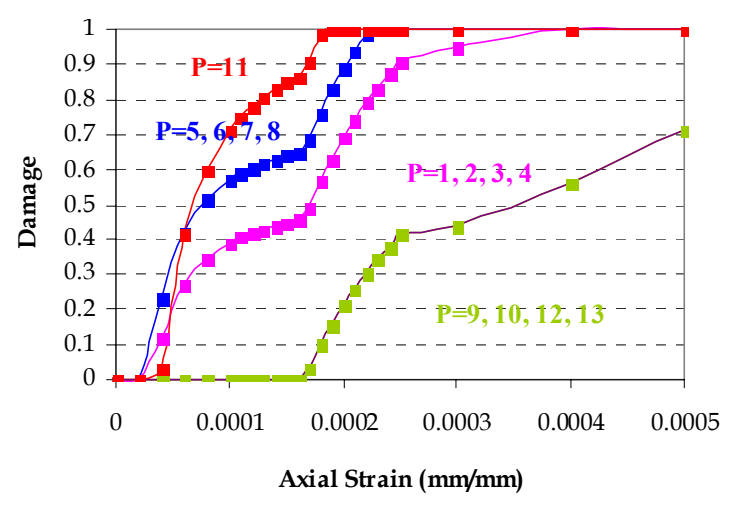

Fig. 6: Damage values on micro-planes
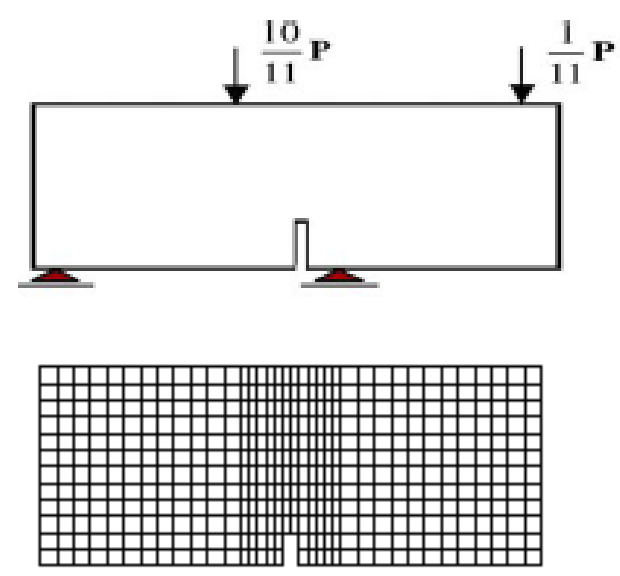

Fig. 7: Single-edge-notched beam subjected to antisymmetric four-point-shear loading

Finite elements code: Three dimensional finite elements software is developed using the proposed concrete model as an alternative nonlinear material analyzing. The source of this software has been developed in previous author's studies ${ }^{[2]}$. This new software can performs crack/damage analysis of 3D concrete structures under static/dynamic and monotonic/cyclic loadings. The operating sequence of proposed code for nonlinear static analysis is illustrated in Fig. 1.

Validity assessment: Validity of the proposed code is investigated here through analysis of a few standard benchmark examples with the model and comparison of its obtained results with the published paper results.

Three-point-bending test: For the first example, the three-point-bending test of Bažant and Pfeiffer is considered $^{[3]}$. Figure 2 shows the geometry and finite element meshes for this test.

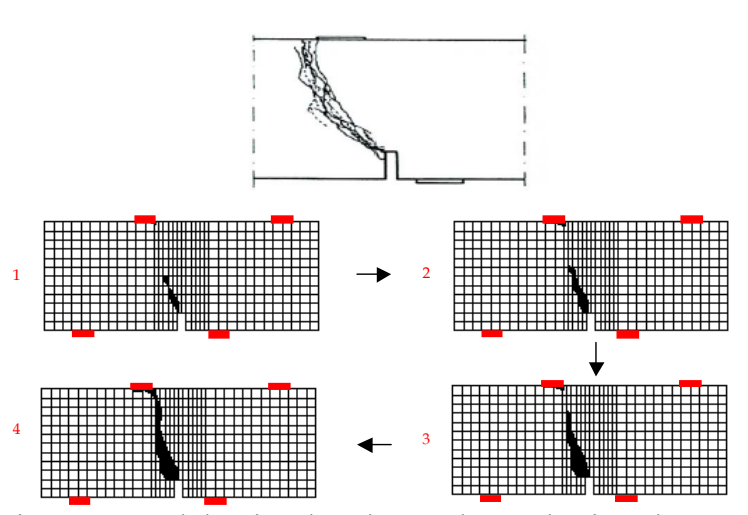

Fig. 8: Model simulated crack path in the antisymmetric four-point-shear test

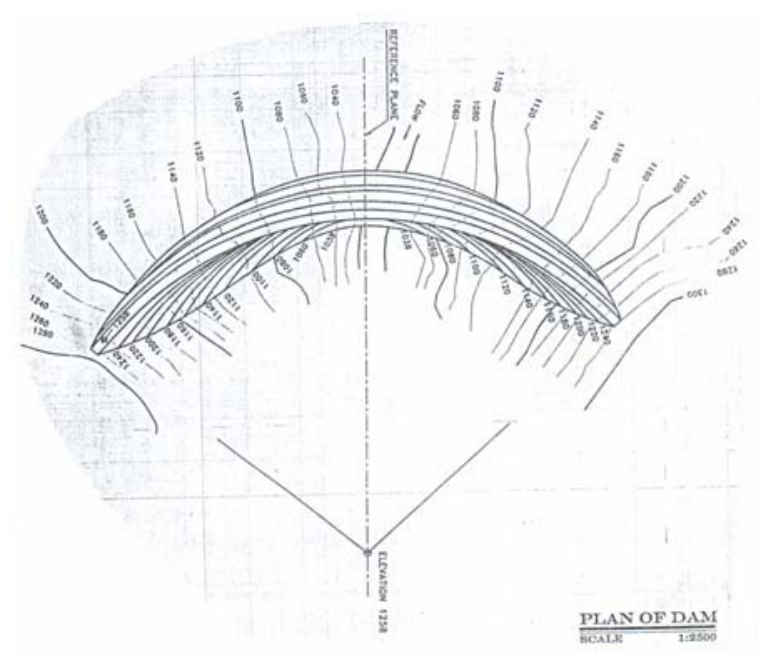

Fig. 9: Arch dam plan

The tensile strength of concrete and elastic modulus are assumed as $\mathrm{f}^{\prime}{ }_{\mathrm{t}}=2.8 \mathrm{Mpa}$ and $\mathrm{E}=27500 \mathrm{Mpa}$. For modeling, 428 three dimensional eight noded brick elements and 958 nodes are used. The simulated crack growth path with the proposed model is illustrated in Fig. 3. As it can be seen from this picture the damaged region of specimen is coincident with experimental observations. In Fig. 4, the obtained load-displacement curve of proposed model and that of belongs to reference ${ }^{[3]}$ are compared. There is a good agreement between the obtained and existed results.

This example shows the ability of the proposed code in simulating mode I fracture of concrete in 3D concrete structures. Furthermore, because of the specific formulation of damages on micro-planes, the suggested model is able to predict the direction of crack line in each integration points. This can be observed in Fig. 5, in which the damaged micro-planes in integration point number 681 are illustrated (orange 


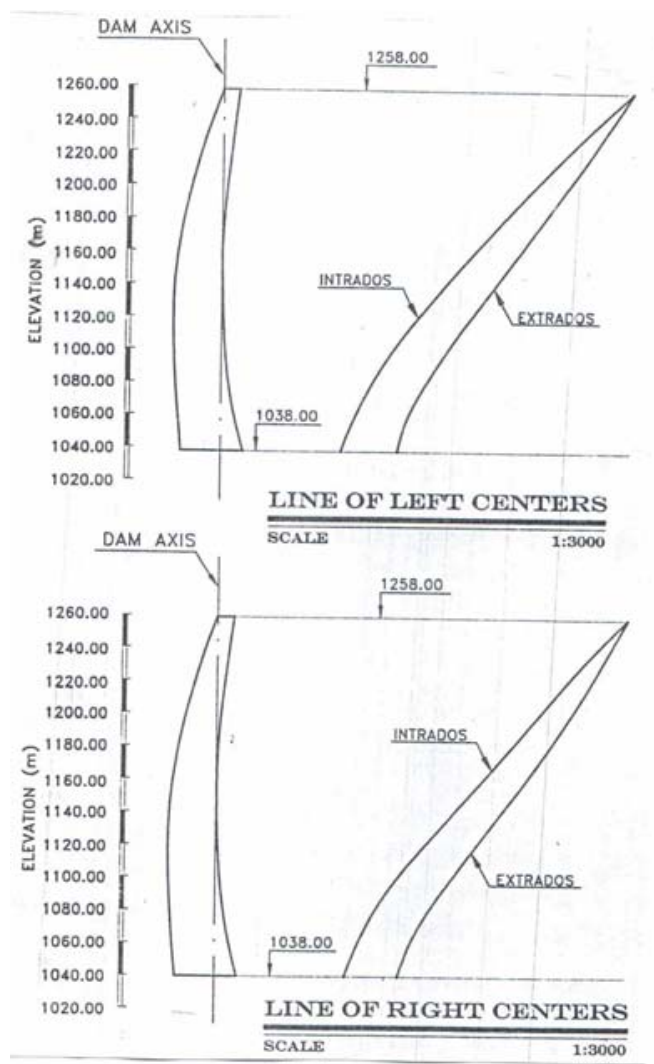

Fig. 10: Crown cantilever and lines of the centers of dam

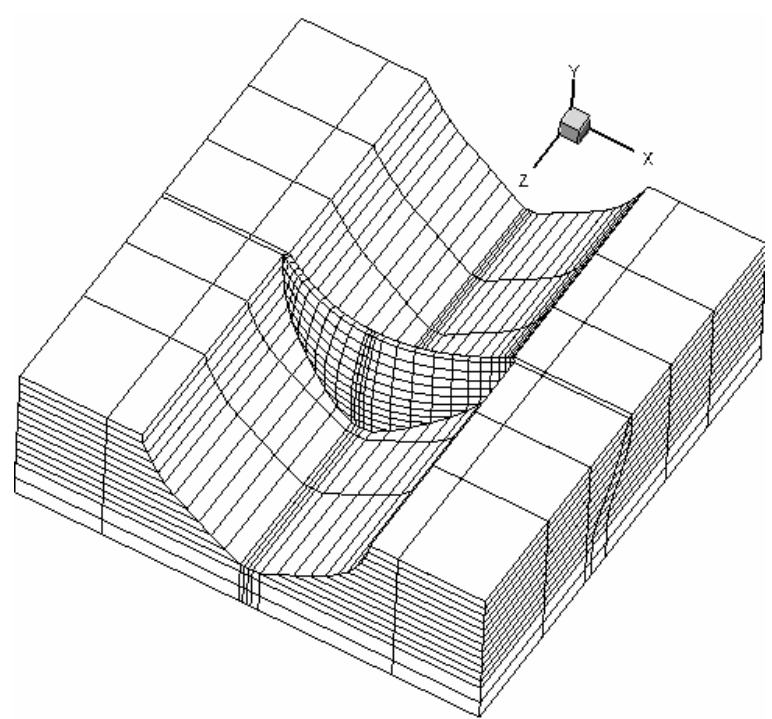

Fig. 11:Finite elements mesh of the arch dam

color planes). On these planes only a tensile strain exist (load condition II) and mode I fracture has occurred on them. Comparison between damage values obtained

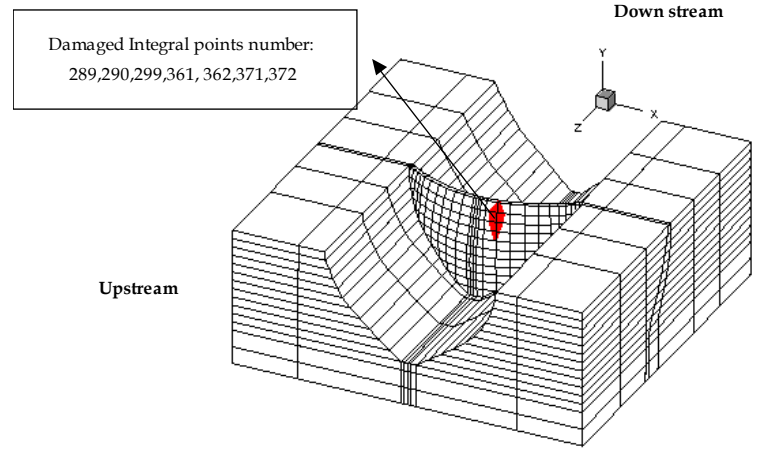

Fig. 12: Damaged parts of the arch dam monitored by proposed model

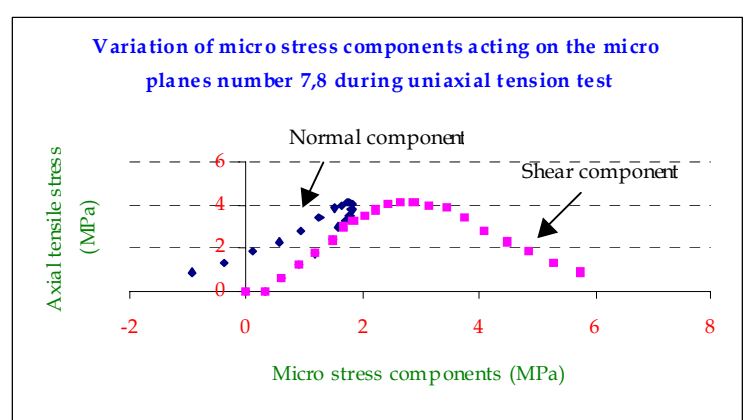

Fig. 13: Stress paths on micro-planes number 7,8 of integral point number 371

with the proposed code on various micro-planes in integration point 681 is shown in Fig. 6.

Anti-symmetric four-point-shear test: To show how the model is also able to predict a crack with a curved path, a notched specimen, subjected to an antisymmetric four-point-shear loading, is considered. The thickness of the beam is $38 \mathrm{~mm}$. A mesh of 428 three dimensional brick elements is used (Fig. 7). The material parameters are $\mathrm{E}=27500 \mathrm{Mpa}$ and $v=0.20$.

When the anti-symmetric load is applied, a crack starts from the left corner of the notch and grows upwards to the left side of the loading platen. Figure 8 shows the crack path in the specimen obtained from the proposed model and the actual monitored crack path obtained from experiment. As it is obvious from this picture, the proposed model can simulate crack line well. Mode II fracture has occurred on micro-planes number 5,6,7,8 (Fig. 5-blue planes) due to combination of tensile and shear stresses (load condition V) in damaged integration points.

Arch dam crack analysis: For showing the abilities of the proposed model in the applicable examples, a 
double curvature arch dam is selected. This dam is in the feasibility stage of consulting studies in Dez-Ab consulting engineering company and located in the south-west of Iran near to Aligoodarz city.

Dam geometry: Geometry of the dam including Plan and profiles of the crown cantilever and lines of the centers are shown in Fig. 9 and 10. Dam has $220 \mathrm{~m}$ height and the length of its crown is about $430 \mathrm{~m}$.

Material library: Elastic modulus and Poisson's ratio of plain concrete of the dam are considered as $E_{c}=30000 \mathrm{MPa}$ and $v_{\mathrm{c}}=0.20$. Mass density of concrete is depicted as $\rho=2400 \mathrm{~kg} \mathrm{~m}^{-3}$. Elastic modulus of rock mass is selected as $E_{R}=20000 \mathrm{MPa}$.

Finite elements mesh: Model discretization of the arch dam used in the analysis is shown in Fig. 11. Total of 652 three dimensional brick elements and 1146 node are used for finite elements calculations.

Static analysis: The proposed arch dam is analyzed by the presented code under its weight and hydrostatic pressure of the reservoir. Damaged partitions of dam body which is monitored by damage parameter of proposed concrete model (damage value $\omega \approx 1.0$ ) is illustrated in Fig. 12.

From outputs of the software, it can be observed that damage or crack in the all designated integral points in Fig. 12 occur on micro-planes number 7, 8 which there exists tensile and shear micro-planes stress components (mode II fracture). This is shown that the combination of tensile and shear stresses (load condition V) has the greatest damage effects in our argued arch dam under the effect of its weight and pressure of the reservoir. The stress paths of tensile and shear micro-planes components on micro-planes number 7, 8 from initial loading stage up to the failure is depicted in Fig. 13.

\section{CONCLUSION}

The proposed 3D finite elements software which mobilized with proposed author's micro-planes damage based concrete model ${ }^{[1]}$, has the capability of crack/damage analysis of actual concrete structures. This presented code not only can determine the direction of crack line in three dimensional spaces, but also assigns which loading conditions are responsible to crack event. This ability rises from the specific unique formulation of damage on each micro-plane at any integration points. Each integration points in the proposed software equipped with 26 micro-planes, on each of them a specific damage value computed during analysis according to its own loading conditions.

\section{REFERENCES}

1. Labibzadeh, M. and S.A. Sadrnejad, 2006. Mesoscopic damage based model for plane concrete under static and dynamic loadings. Am. J. Appl. Sci., 3: 2011-2019.

2. Labibzadeh, M. and S.A. Sadrnejad, 2006. Dynamic solution code for structural analysis upon joint element. J. Computer Sci., 2: 401-409.

3. Bažant Z. P., Giovanni, D. L., 2004. Nonlocal micro-planes model with strain-softening yield limits. Intl. J. Solids and Structures, 41: 7209-7240. 